\title{
Macroinvertebrados acuáticos presentes en dietas de peces de la cuenca del río Hacha (Caquetá, Colombia)
}

\author{
Jhonatan Gutiérrez-Garaviz ${ }^{1, *}$, Marlon Peláez-Rodríguez², Hernando Ovalle-Serrano³ \\ ${ }^{1}$ Calidad y preservación de ecosistemas acuáticos, Universidad de la Amazonia, Caquetá, Colombia; \\ grupo de estudios en recursos hidrobiológicos continentales, Universidad del Cauca, Cauca, Colombia \\ ${ }^{2}$ Calidad y preservación de ecosistemas acuáticos, Universidad de la Amazonia, Caquetá, Colombia \\ ${ }^{3}$ Centro de Investigación Ambiental-Corporación Autónoma de Cundinamarca, Colombia
}

\begin{abstract}
Resumen
Los macroinvertebrados acuáticos (MA) exhiben una amplia variedad de funciones en los ecosistemas acuáticos (detritívoros, depredadores y alimento) lo cual mantiene el equilibrio en el los flujos de materia y energía. En el presente estudio se confirma la importancia trófica de MA en la dieta de peces de sistemas lóticos. El estudio se desarrolló en la cuenca del río Hacha (Caquetá, Colombia) en 6 estaciones de muestreo; se realizó la captura de peces para el análisis de sus contenidos estomacales. Adicional se tomaron muestras de MA como material de referencia para comparar con los encontrados en los contenidos estomacales de los peces. Para el análisis de datos, se aplicaron tres índices de diversidad en los MA; respecto a los contenidos estomacales en peces se estimó el porcentaje de frecuencia de ocurrencia (\%FO) y el porcentaje de dominancia (\%D) y se evaluó el solapamiento de nicho mediante un clúster con distancia euclidiana. Las estaciones de la cuenca alta y media presentaron los mayores valores de diversidad de MA. Respecto a los peces, la familia Characidae agrupó la mayor riqueza de especies; Astyanax abramis fue el taxón más representativo en distribución y abundancia. En términos de hábitos alimenticios, se identificaron especies de peces generalistas que consumen MA y especialistas que pueden o no consumir. Characidium zebra y Astyanax abramis, presentan una fuerte relación con el consumo de MA. Leporinus frieredici y Leporinus y-ophorus fueron las especies con mayor solapamiento de nicho. (C) 2016. Acad. Colomb. Cienc. Ex. Fis. Nat.
\end{abstract}

Palabras clave: Diversidad; Hábitos alimenticios; Peces; Invertívora; Solapamiento de nicho.

Aquatic macroinvertebrate present in fish diets of the basin of Hacha river (Caquetá, Colombia)

\begin{abstract}
Aquatic macroinvertebrates (MA) are vital to the daily health of their related habitats, as detritivores, predators, and prey. In this study we demonstrate the trophic importance of MA's, as prey items to fishes in the river basin Hacha (Florencia-Caquetá) by analyzing their stomach contents. Six sampling stations were defined taking into account upper, middle and lower part of the basin; Field samples of MA as reference material and fish were collected were captured 3 arts used traditional fishing. For data analisys, three indices of diversity were calculated for MA; regarding fish stomach contents was estimated percentage frequency of occurrence $(\% \mathrm{FO})$ and the percentage of dominance (\%D), plotting their values; niche overlap was also evaluated using a cluster with euclidean distance. Stations 1 , 2, 3 and 4 showed the best values of diversity of MA. The Characidae family has the highest species richness of Characiformes fish, being Astyanax abramis with better distribution and abundance. Generalist species that consume aquatic macroinvertebrates and specialist species that may or may not consume aquatic macroinvertebrates were identified. Characidium abramis zebra and Astyanax, are more related to consumption MA. Leporinus friderici and Leporinus y-ophorus were those with high niche overlap. (C) 2016. Acad. Colomb. Cienc. Ex. Fis. Nat.
\end{abstract}

Key Words: Diversity; Eating habits; Fish; Invertivora; Niche overlap.

\section{Introducción}

Los macroinvertebrados acuáticos (MA) y peces se consideran las principales comunidades de animales que se desarrollan en los ecosistemas de agua dulce (Roldán, 1992), encontrando una gran diversidad de especies en la región Neotropical. Los MA en su mayoría insectos, son aquellos organismos que se pueden ver a simple vista; Roldán \& Ramírez (2008) los clasifican a partir de un tamaño mayor a 0,5 mm de longitud, habitando en los ecosistemas lóticos, piedras, troncos, hojarasca, enterrados en el fondo, adheridos a vegetación y nadando libremente dentro del agua o sobre la superficie (Roldán, Posada \& Gutiérrez, 2001).

\footnotetext{
*Correspondencia:

Jhonatan Gutiérrez-Garaviz, jhotas24@gmail.com

Recibido: 25 de febrero de 2016

Aceptado: 20 de junio de 2016
} 
Los peces son los principales vertebrados existentes en los ecosistemas acuáticos; la presencia de esta comunidad define las redes tróficas (Ramírez \& Viña, 1998), incidiendo sobre la estructura del ecosistema (Carpenter, et al., 1987; Quirós \& Boveri, 1999; Meijer, De Boois, Scheffer, Portielje \& Hosper, 1999; Kasprzak, Benndorf, Mehner \& Koschel, 2002), cuyos efectos dependen de la selectividad del alimento, estructura demográfica, distribución espacial, dominancia de especies (Roche \& Rocha, 2005) y solapamiento de nichos. Así mismo, sus hábitos alimenticios permiten conocer el rol trófico que puede tener con las diferentes comunidades acuáticas (Hyslop, 1980; Barros, 2004), en términos de consumo y disponibilidad de alimento en el medio (Muñoz, Romaní, Rodríguez-Capítulo, González \& García-Berthou, 2009). Estos hábitos se pueden clasificar a partir de los ítems ingeridos, identificando así especies omnívoras, planctívoras, detritívoras, iliófagas, piscívoras, insectívoras, herbívoras e invertivoras (Hahn, Fugi, De Almeida, Russo \& Loureiro, 1997).

Las especies ícticas que consumen macroinvertebrados acuáticos se clasifican como invertívoras (Ortaz, Martín \& López-Ordaz, 2011); estas especies moldean constantemente la estructura de la comunidad presa (Flecker, 1992; Taphorn, 1992), incidiendo como variable biológica sobre su abundancia y riqueza.

Una de las metodologías que se emplea para establecer la relación trófica entre MA y peces es el análisis de contenidos estomacales (Hyslop, 1980; Amundsen, Gabler \& Staldvik, 1996; Renó, Bornatowski \& Simões, 2012). En Colombia, Sánchez, Galvis \& Victoriano (2003), Aten et al. (2005), Maldonado-Ocampo, et al. (2005), Galvis, et al. (2006), Torres, Roldán, Asprilla \& Rivas (2006), Zamudio, Urbano, Maldonado-Ocampo, Bogota-Gregory \& Cortes-Millan (2008), Olaya-Nieto, Soto-Fernández \& Barrera-Chica (2009), Olaya-Nieto, Arellano-Padilla \& Martínez-González (2012), utilizaron diferentes métodos para el análisis de contenidos estomacales, identificando a la comunidad de MA como ítem alimenticio importante.

En la presente investigación se identificaron las especies ícticas que se alimentan de macroinvertebrados acuáticos y cuáles son los taxones consumidos, a partir de la proporción y composición en sus contenidos estomacales.

\section{Metodología}

Área de estudio: El estudio se llevó a cabo en la cuenca alta (estación 1 y 2), media (estación 3 y 4) y baja (estación 5 y 6) del río Hacha (Florencia-Caquetá), con un rango altitudinal que va desde los 919 msnm hasta los 253 msnm, en la región andino-amazónica del departamento del Caquetá; entre las coordenadas de referencia N $1^{0} 47^{\prime} 16,4^{\prime \prime}$ - W 750 39'13,8'" y N $1^{0} 35^{\prime} 39,1^{\prime \prime}$ - W $75^{0} 35^{\prime}$ 42,1'” (Figura 1). En términos de paisaje se identificaron bosque, agricultura, pastoreo y asentamientos humanos, principalmente en la estación 5 relacionada con la cabecera municipal de Florencia; en la mayoría de estaciones se identificó vegetación de tipo arbórea, arbustiva, herbácea y pasturas, a excepción de la estación 6 donde dominan los pastos, con alta fragmentación del bosque. En la cuenca alta y medía se registró un 90 a 70\% de vegetación riparia, disminuyendo en la cuenca baja hasta un 20\% (estación 6). El agua se observó transparente en las primeras 4 estaciones, aumentando la turbidez en la estación 5 y 6, cuyas características están dadas por la geomorfología del lecho del río, dominando el sustrato rocoso en la cuenca alta y media, y fino en la cuenca baja.

Fase de campo y laboratorio: El tiempo de muestreo abarco los meses de mayo a diciembre de 2014 y enero de 2015. En las seis estaciones que se trabajaron, la información biológica se colecto en un tramo de 100 m de longitud, realizando una réplica por punto. Para la colecta de MA, se utilizó una red surber de marco de $30 \mathrm{~cm}^{2}$ con poro de malla de 150 micras y se tuvo en cuenta la composición del sustrato en el lecho río para así abarcar los diferentes microhábitats disponibles, y los sitios donde se capturaron peces. Posteriormente, el material fue preservado en etanol al 95\% y llevado al laboratorio para conteo e identificación de los diferentes taxones con la ayuda de guías y claves taxonómicas de McCafferty (1981), Roldán (1988), Klemm (1995), Epler (1995, 1996), Merrit, Cummins \& Berg (2008), Voshell (2002), Pescador, Ramussen \& Harris (2002), Pescador \& Barton (2004), Heckman (2008), Domínguez \& Fernández (2009), Springer, Ramírez \& Hanson (2010).

Cuenca hidrográfica del río Hacha (Florencia, Caquetá)

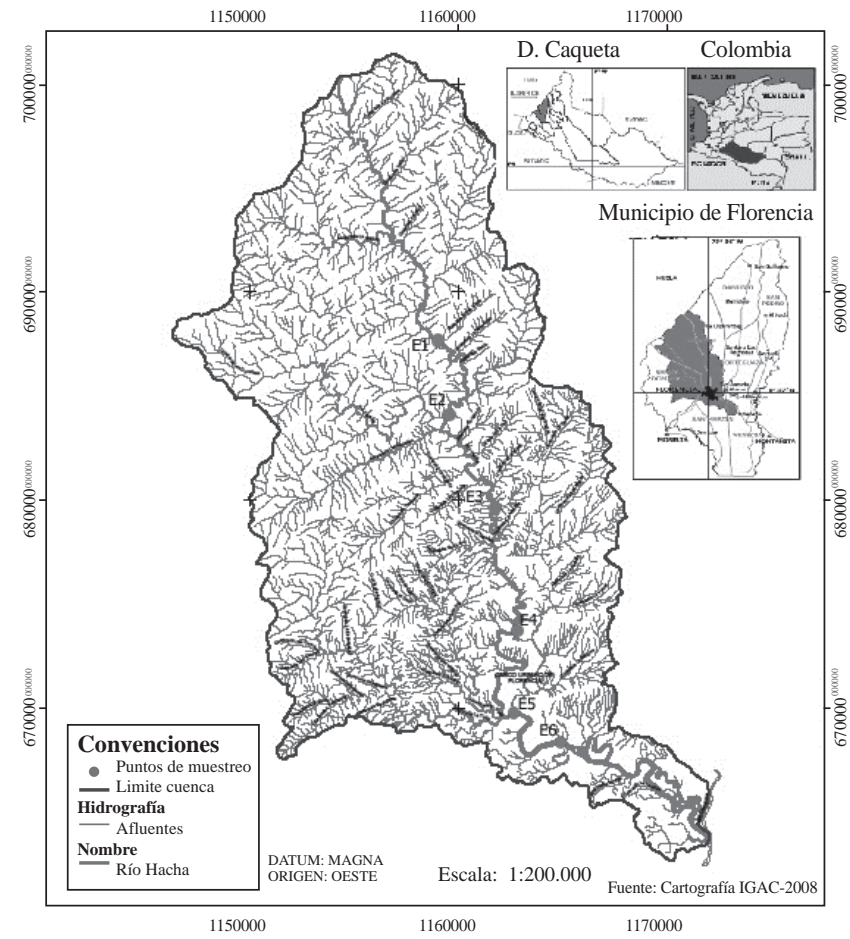

Figura 1. Mapa Cuenca del Río Hacha. Se identifican las estaciones de muestreo en círculos.

Fuente: Remicio (2014), modificado por los autores. 
Los peces fueron capturados utilizando tres artes tradicionales, chile, anzuelo y caretaje nocturno manual. Con el chile se realizaron en promedio 50 lances aleatorios sobre el tramo de estudio. Los anzuelos se fijaron en varas ancladas en los dos márgenes del río. El caretaje se realizó en la noche, utilizando linterna, pistola y una malla de mano para la captura de especímenes, sumergiéndose en el agua y buscando en los diferentes sustratos presentes (se empleó principalmente para la captura de individuos de Characidium zebra). Los individuos cuya longitud no superaron los 50 $\mathrm{mm}$ se preservaron en frascos plásticos de $500 \mathrm{~cm}^{3}$ con solución Transeau; los peces con talla mayor se inyectaron por el ano con formol al $10 \%$ y se guardaron en bolsas herméticas. Posteriormente se llevaron al laboratorio para su identificación utilizando las guías y claves taxonómicas de Géry (1977), Galvis, et al. (1997, 2006), Mojica (1999), Maldonado-Ocampo, et al. (2005), Gregory \& Maldonado-Ocampo (2006), Román-Valencia (2007), Rubio (2007) y Sanabria, Daza \& Beltrán (2007).

Para analizar los contenidos estomacales en peces se tuvo en cuenta las recomendaciones expuestas por Marrero (1994) y Muñoz, et al. (2009), diseccionando los especímenes y retirando el estómago; el contenido se depositó en una caja de Petri con alcohol al 70\%. Los restos de MA fueron separados y comparados con el material de referencia colectado en campo.

Análisis de datos: Para la comunidad de MA se determinaron los índices de diversidad de Shannon-Weaver, Pielou y la riqueza específica, en cada una de las estaciones de estudio, utilizando el programa Past V2.1 (Hammer, Harper \& Ryan, 2002).

Respecto a la estimación de los ítems alimenticios en los estómagos examinados, se utilizó el porcentaje de frecuencia de ocurrencia $(\% \mathbf{F O})$, quien relaciona el número de estómagos con el ítem alimenticio específico, respecto al número total de estómagos registrados (Hyslop, 1980). La fórmula es la siguiente:

Dónde:

$$
\% \mathrm{FO}=\frac{\mathrm{j}}{\mathrm{p}} \times 100
$$

$j$ : estómagos con ítem alimenticio específico.

$p$ : total de estómagos con ítem registrados.

También se tuvo en cuenta la propuesta de análisis de ítems alimenticios en peces evaluado por Bennemann, Cassatti \& Cristiane de Oliveira (2006), expresado como dominancia relativa, haciendo referencia al número de veces en que un ítem específico ocupa la mayor parte del contenido estomacal en función del número total de estómagos analizados.

Dónde:

$$
\% \mathrm{D}=\frac{\mathrm{j}}{\mathrm{P}} \times 100
$$

$j$ : estómagos con ítem alimenticio específico.

$p$ : total de estómagos con ítem registrados.

Los datos obtenidos en los estimativos anteriores fueron graficados teniendo en cuenta la metodología de Costello (1990), ubicando la Dominancia (\%) en el eje Y y la Frecuencia de Ocurrencia (\%) en el eje X. Bennemann, et al. (2006) modifica la ecuación de Costello (1990) sustituyendo Peso (\%) por Dominancia (\%). Esta gráfica permite visualizar la importancia de cada ítems alimenticio. Las gráficas se realizaron con el programa "R V3.3.2 utilizando la librería plot”.

Además se evaluó el solapamiento de nicho entre peces a través de la distancia euclidiana, teniendo en cuenta los valores de dominancia representativos en las especies que presentan MA como ítems significativo. Para este análisis se utilizó el programa "Past V2.1" (Hammer, et al., 2002).

\section{Resultados}

Macroinvertebrados acuáticos: Se recolectaron en total 5 phylum, 6 clases, 15 órdenes, 41 familias, 63 géneros y 971 organismos, donde la clase Insecta fue la categoría taxonómica con mayor abundancia de individuos (Tabla 1). A nivel de órdenes, Trichoptera, presento la mayor dominancia de géneros en las seis estaciones de estudio, seguido de Diptera y Coleoptera. En términos de diversidad, las estaciones 1, 2, 3, 4 tienen una mayor riqueza, diversidad y mejor distribución de taxones en el medio acuático, a diferencia de las estaciones 5 y 6, cuyos valores fueron los más bajos (Tabla 2).

Peces: se colectaron 2 órdenes, 9 familias, 24 especies y 752 individuos (Tabla 3). El orden Characiformes tiene la mayor riqueza de especies, al igual la familia Characidae, siendo la especie Astyanax abramis el taxón más abundante y con mayor distribución a lo largo de la cuenca del río Hacha. Loricaridae es una familia que también presento igual número de especies que Characidae, pero su individuos son bentónicos detritívoros y no tienen una relación fuerte con los MA, al revisar algunos contenidos estomacales en el presente estudio.

Análisis de contenidos estomacales: teniendo en cuenta la Tabla 4 se observa que la mitad aproximada de taxones del orden Characiformes presentan macroinvertebrados acuáticos como ítem alimenticio representativo. En el caso de los Siluriformes, Pimelodella cf. cristata y Pimelodus blochii son las únicas dos especies que se relacionan con el ítem de estudio. De los 24 taxones pre-seleccionados se escogen solo 9, teniendo en cuenta una mayor presencia de macroinvertebrados acuáticos en los contenidos estomacales (Tabla 4). Astyanax abramis es la especie con mayor número de ítems alimenticios.

Gráficas de Costello: al hacer la representación por el método gráfico de Costello (1990), (Figura 2), se identificaron claramente 2 grupos tróficos: Especies generalistas omnívoras que contienen MA, Astyanax abramis (familias Baetidae, Chironomidae y Libellulidae) y Leporinus friederici (Chironomidae). En el caso de Pimelodella cf. cristata, se observó una tendencia hacia el grupo trófico especialista de chironómidos (especie invertívora). El segundo grupo son especies especialistas con dominancia del algún ítem particular, que pueden o no contener MA. Pimelodus blochii, Hemibricon sp. Brycoamericus sp. y Creagrutus cochoui, se alimentaron principalmente de 
Tabla 1. Composición macroinvertebrados acuáticos. Se muestran las abundancias de los diferentes taxones recolectados en campo. No determinado (N.D).

\begin{tabular}{|c|c|c|c|c|c|c|c|c|c|c|}
\hline PHYLLUM & CLASE & ORDEN & FAMILIA & GÉNERO & E1 & E2 & E3 & E4 & E5 & E6 \\
\hline \multirow[t]{2}{*}{ Annelida } & Oligochaeta & Haplotaxida & Naididae & N.D & - & - & - & 6 & 84 & 26 \\
\hline & Hirudinea & Rhynchobdellida & Glossiphoniidae & Helobdella spp. & - & - & - & - & - & 3 \\
\hline \multirow[t]{57}{*}{ Arthropoda } & Arachnoidea & Acari & N.D & N.D & - & - & - & 1 & - & 4 \\
\hline & Insecta & Coleoptera & Dytiscidae & cf. Celina & 1 & - & - & - & - & - \\
\hline & & & Elmidae & Phanocerus spp. & 3 & 1 & - & - & - & - \\
\hline & & & & N.D 1 & 6 & 2 & 2 & 9 & 2 & - \\
\hline & & & & N.D 2 & 2 & - & - & - & - & - \\
\hline & & & & Heterelmis spp. & 14 & 7 & 22 & 22 & - & 4 \\
\hline & & & & Hexanchorus spp. & 2 & 1 & 1 & - & - & - \\
\hline & & & & Macrelmis spp. & 3 & 2 & 1 & 5 & - & - \\
\hline & & & & Cylloepus spp. & - & - & 2 & 3 & - & - \\
\hline & & & & Microcylloepus spp. & 2 & - & - & - & - & - \\
\hline & & & & Pseudodisersus spp. & - & 1 & 3 & 3 & - & - \\
\hline & & & Psephenidae & N.D & - & - & - & 2 & - & - \\
\hline & & & Ptilodactylidae & Anchytarsus spp. & 4 & 8 & 1 & 4 & - & - \\
\hline & & Diptera & Blephariceriidae & $N . D$ & - & 1 & 12 & 3 & - & - \\
\hline & & & Ceratopogonidae & Atrichopogon spp. & - & - & - & 2 & 3 & - \\
\hline & & & Chironomidae & N.D1 & 24 & 16 & 12 & 33 & 95 & 56 \\
\hline & & & & N.D2 & 7 & 1 & 7 & 8 & - & 2 \\
\hline & & & & N.D3 & - & - & 2 & 6 & - & - \\
\hline & & & Empididae & Hemerodromia spp. & 4 & - & 1 & 1 & 2 & - \\
\hline & & & Simuliidae & Simulium spp. & 1 & 1 & - & - & 2 & - \\
\hline & & & Psychodidae & Pericoma spp. & - & - & - & - & 2 & 1 \\
\hline & & & & Psychoda spp. & - & - & - & - & - & 2 \\
\hline & & & Tabanidae & N.D & - & - & - & - & 1 & 1 \\
\hline & & & Tipulidae & Hexatoma spp. & - & - & - & 1 & - & - \\
\hline & & & & Molophilus spp. & - & 2 & - & - & - & - \\
\hline & & Ephemeroptera & Baetidae & Camelobaetidius spp. & 9 & 10 & 7 & 14 & - & - \\
\hline & & & & Baetodes spp. & 4 & 1 & 1 & 1 & 5 & \\
\hline & & & & N.D & - & - & - & - & - & 1 \\
\hline & & & Leptophlebiidae & Thraulodes spp. & 5 & 3 & 1 & 11 & 5 & \\
\hline & & & & Terpides spp. & - & - & - & 1 & - & - \\
\hline & & & & Traverella spp. & 4 & - & 2 & 1 & - & - \\
\hline & & & Leptohyphidae & Leptohyphes spp. & 16 & 4 & 2 & 25 & 7 & - \\
\hline & & & & Tricorythodes spp. & 1 & - & 1 & 8 & - & - \\
\hline & & & Oligoneuriidae & Lachlania spp. & - & - & 2 & - & - & - \\
\hline & & Hemiptera & Naucoridae & Cryphocricos spp. & 3 & - & - & 1 & - & - \\
\hline & & & & Limnocoris spp. & - & - & - & 1 & 4 & - \\
\hline & & & Veliidae & Rhagovelia spp. & - & - & 9 & 5 & 1 & - \\
\hline & & Lepidoptera & N.D & N.D & - & - & - & 1 & - & - \\
\hline & & Megaloptera & Corydalidae & Corydalus spp. & - & 2 & 2 & 1 & - & - \\
\hline & & Odonata & Gomphidae & N.D & - & 1 & - & - & - & - \\
\hline & & & Libellulidae & N.D & 4 & - & - & 1 & 1 & 10 \\
\hline & & & Calopterygidae & Hetaerina spp. & - & - & - & - & - & 2 \\
\hline & & & Coenagrionidae & Enallagma spp. & - & - & - & - & - & 1 \\
\hline & & Plecoptera & Perlidae & Anacroneuria spp. & 8 & 2 & 4 & 13 & - & - \\
\hline & & Trichoptera & Calamoceratidae & Phylloicus spp. & - & - & 2 & - & - & - \\
\hline & & & Glossosomatidae & Mortoniella spp. & 13 & 1 & - & - & 1 & - \\
\hline & & & Leptoceridae & Atanatolica spp. & - & - & 4 & - & - & - \\
\hline & & & & Grumichella spp. & 43 & - & - & - & - & - \\
\hline & & & & N.D & - & - & 1 & 1 & - & - \\
\hline & & & Hydrobiosidae & Neoatopsyche spp. & - & - & - & 1 & - & - \\
\hline & & & Helicopsychidae & Helicopsyche spp. & 2 & - & - & - & - & - \\
\hline & & & Hydropsychidae & Leptonema spp. & 6 & - & - & 5 & - & - \\
\hline & & & & Smicridea spp. & 25 & 2 & 3 & 12 & 2 & 8 \\
\hline & & & Hydroptilidae & N.D & 7 & 1 & 1 & - & 2 & - \\
\hline & & & & Ochrotrichia spp. & 2 & - & - & - & - & - \\
\hline & & & Philopotamidae & Chimarra spp. & 3 & 1 & 2 & 2 & - & - \\
\hline & & & Polycentropodidae & Cyrnellus spp. & - & 3 & - & - & - & - \\
\hline \multirow[t]{2}{*}{ Mollusca } & & & Lymnaeidae & Lymnaea spp. & - & - & - & - & - & 2 \\
\hline & & Mesogastropoda & Hydrobiidae & N.D & - & - & - & 1 & - & - \\
\hline Nemathomorpha & N.D & N.D & N.D & N.D & 1 & - & - & - & - & - \\
\hline Plathelminthes & Turbelaria & Tricladida & Planariidae & Dugesia spp. & - & - & - & - & 1 & - \\
\hline
\end{tabular}


Tabla 2. Índices de diversidad, macroinvertebrados acuáticos. Índice de diversidad de Shannon-Weaver (H’), equitatividad de Pielou (J’).

\begin{tabular}{lcccccc}
\hline ÍNDICES & E1 & E2 & E3 & E4 & E5 & E6 \\
\hline Riqueza & 31 & 24 & 28 & 36 & 18 & 15 \\
Diversidad $\left(\boldsymbol{H}^{\prime}\right)$ & 2,9260 & 2,7080 & 2,8390 & 3,0050 & 1,5220 & 1,8060 \\
Equitatividad $(\boldsymbol{J})$ & 0,8522 & 0,8521 & 0,8518 & 0,8385 & 0,5267 & 0,6670 \\
\hline
\end{tabular}

Tabla 3. Composición íctica. Se muestran los diferentes taxones colectados con la metodología propuesta.

\begin{tabular}{|c|c|c|c|c|c|c|c|c|}
\hline ORDEN & FAMILIA & ESPECIE & E1 & E2 & E3 & E4 & E5 & E6 \\
\hline \multirow[t]{15}{*}{ Characiformes } & Anostomidae & Leporinus friderici (Bloch, 1974) & - & - & - & - & - & 42 \\
\hline & & Leporinus y-ophorus (Eigenmann, 1922 ) & - & - & - & - & - & 38 \\
\hline & & Leporinus striatus (Kner, 1858 ) IDEM A LO ANTERIOR & - & - & 1 & 1 & - & - \\
\hline & Characidae & Astyanax abramis (Jenyns, 1842) & - & 35 & 56 & 57 & 66 & 36 \\
\hline & & Astyanax fasciatus (Cuvier, 1819) & - & - & - & - & 4 & - \\
\hline & & Creagrutus cochui (Géry, 1964) & - & 2 & - & 2 & - & - \\
\hline & & Bryconamericus sp. ( Eigenmann, McAtee and Ward, 1907) & - & - & - & 7 & - & - \\
\hline & & Hemibrycon sp. (Günter 1864) & - & 56 & 44 & 55 & - & - \\
\hline & & Knodus sp. (Eigenmann 1911) & - & - & 1 & - & - & - \\
\hline & & Tetragonopterus argenteus (Cuvier, 1816) & - & - & - & - & - & 12 \\
\hline & Crenuchidae & Characidium zebra (Eigenmann, 1909) & 7 & 8 & 10 & - & - & - \\
\hline & Curimatidae & Steindachnerina bimaculata (Steindachner, 1876) & - & - & - & - & 6 & - \\
\hline & Parodontidae & Parodon cf. Pongoensis (Allen 1942 en Eigenmann \& Allen, 1942) & - & - & - & - & 2 & - \\
\hline & & Parodon sp. (Cuvier and Valenciennes,1850) & - & - & - & 14 & - & - \\
\hline & Prochilodontidae & Prochilodus nigricans (Spix \& Agassiz, 1829) & - & - & - & 1 & 5 & 5 \\
\hline \multirow[t]{9}{*}{ Siluriformes } & Heptapteridae & Pimelodella cf. Cristata (Müller \& Troschel, 1849) & - & - & - & - & - & 47 \\
\hline & Loricariidae & Ancistrus sp1. (Kner, 1854) & - & 12 & 9 & 5 & 2 & - \\
\hline & & Ancistrus sp2. (Kner, 1854) & - & - & - & 2 & - & - \\
\hline & & Cordylancistrus sp. ( Isbrücker, 1980) & 15 & 2 & & 6 & 1 & - \\
\hline & & Chaetostoma sp. (Tschudi, 1846) & 6 & - & 3 & 1 & - & - \\
\hline & & Hypostomus plecostomus (Linnaeus, 1758) & - & - & - & 3 & 7 & 1 \\
\hline & & Rineloricaria sp1. (Bleeker, 1862 ) & - & - & - & - & 4 & - \\
\hline & & Rineloricaria sp2. (Bleeker, 1862 ) & - & - & - & - & 8 & - \\
\hline & Pimelodidae & Pimelodus blochii (Cuvier \& Valenciennes, 1840) & - & - & - & - & - & 45 \\
\hline
\end{tabular}

hormigas (especies insectívoras que consumen individuos de la familia Formicidae), encontrando MA frecuentes pero no dominantes. Leporinus y-ophorus es una especie con características detritívoras, pero contiene un valor significativo de MA, al igual que Brycoamericus sp. que consume hormigas (especie insectívora), con un valor importante de quironómidos. Characidium zebra es una especie invertívora que consume principalmente tricópteros de la familia Hydropsychidae, además de otros MA y restos vegetales.

Contrastando los resultados de hábitos alimenticios para cada especie con la literatura se encontró resultados similares, resumidos en la Tabla 5.

Solapamiento de nicho: Leporinus friederici y Leporinus $y$-ophorus fueron las especies con mayor similitud en el consume de items alimenticios (Figura 3). Este agrupamiento está determinado por el consumo de quironómidos, restos vegetales y detritus. También se conformó un segundo grupo con Astyanax abramis y Pimelodella cf. cristata, cuyos ítems de agrupamiento son semillas, hormigas y quironómidos.
Characidium zebra y Brycoamericus sp. fueron las especies que más difieren del grupo; para la primera, su dieta alimenticia está conformada principalmente por MA, respecto a la segunda se caracterizó por consumir hormigas (Formicidae).

\section{Discusión}

Macroinvertebrados acuáticos: la clase Insecta constituye el grupo más numeroso de los Artrópodos, cuyo carácter distintivo es la presencia de estructura articuladas (Masiac, Guilbot \& Albouy, 2006). En el río Hacha, se encontraron principalmente en estados inmaduros (ninfas, larvas), al igual que en los contenidos estomacales, con excepción de los vélidos y algunos coleópteros de la Familia Elmidae en estado adulto.

El dominio de Trichoptera, Diptera y Coleoptera, contrasta con los resultados reportados por López, Gaspar \& Peláez (2012), para la misma cuenca. En los contenidos estomacales (Tabla 4) se identificaron 3 familias de tricópteros que se fijan en sustratos duros, Hidroptylidae y 
Tabla 4. Análisis de contenidos estomacales.

Pimelodus blockii (Rango longitud estándar: $\Delta \mathrm{CP}=13-15,6 ; \mathrm{n}=30$ )

\begin{tabular}{lrrrr}
\hline Ítem alimenticio & FO & \%FO & D & \%D \\
\hline Resto vegetal & 15 & 50,0 & 5 & 16,7 \\
Semilla & 7 & 23,3 & 2 & 6,7 \\
Escama & 16 & 53,3 & 5 & 16,7 \\
Formicidae & 26 & 86,7 & 15 & 50,0 \\
Vertebrado & 3 & 10,0 & 2 & 6,7 \\
\hline Detritus & 1 & 3,3 & 1 & 3,3 \\
Mineral & 9 & 30,0 & - & $<0,1$ \\
Invertebrado & 8 & 26,7 & - & $<0,1$ \\
Chironomidae & 26 & 86,7 & - & $<0,1$ \\
Psychodidae & 5 & 16,7 & - & $<0,1$ \\
Elmidae & 6 & 20,0 & - & $<0,1$ \\
Naididae & 2 & 6,7 & - & $<0,1$ \\
Hidroptylidae & 1 & 3,3 & - & $<0,1$ \\
\hline
\end{tabular}

Pimelodella cf. Cristata (Rango longitud estándar: $\Delta \mathrm{CP}=9,2-12,5 ; \mathrm{n}=30$

\begin{tabular}{lrccc}
\hline Ítem alimenticio & FO & \%FO & D & \%D \\
\hline Semillas & 4 & 13,3 & 5 & 16,7 \\
Restos vegetales & 7 & 23,3 & 9 & 30 \\
Formicidae & 4 & 13,3 & 2 & 6,7 \\
Chironomidae & 21 & 70,0 & 14 & 46,7 \\
Invertebrado & 4 & 13,3 & - & $<0,1$ \\
Detritus & 2 & 6,7 & - & $<0,1$ \\
Vertebrado & 5 & 16,7 & - & $<0,1$ \\
Piedras & 4 & 13,3 & - & $<0,1$ \\
Mineral & 21 & 70,0 & - & $<0,1$ \\
Elmidae & 1 & 10,0 & - & $<0,1$ \\
Escama & 5 & 16,7 & - & $<0,1$ \\
\hline
\end{tabular}

Leporinus friederici (Rango longitud estándar: $\Delta \mathrm{CP}=12-20 ; \mathrm{n}=30$ )

\begin{tabular}{lrrrr}
\hline Ítem alimenticio & FO & \%FO & D & \%D \\
\hline Detritus & 23 & 76,7 & 12 & 40,0 \\
Chironomidae & 23 & 76,7 & 4 & 13,3 \\
Resto vegetal & 24 & 80,0 & 10 & 36,7 \\
Escama & 9 & 30,0 & 3 & 10,0 \\
Mineral & 8 & 26,7 & 1 & 3,3 \\
Formicidae & 4 & 13,3 & - & $<0,1$ \\
Psychodidae & 8 & 26,7 & - & $<0,1$ \\
\hline
\end{tabular}

Leporinus $y$-ophorus (Rango longitud estandar: $\Delta \mathrm{CP}=14-18 ; \mathrm{n}=30$

\begin{tabular}{lrrrr}
\hline Ítem alimenticio & FO & \%FO & D & \%D \\
\hline Detritus & 20 & 66,7 & 16 & 53,3 \\
\hline Chironomidae & 27 & 90,0 & 2 & 6,7 \\
Hydropsychidae & 7 & 23,3 & 1 & 3,3 \\
Resto vegetal & 15 & 50,0 & 11 & 36,7 \\
Acari & 2 & 6,7 & - & $<0,1$ \\
Empididae & 5 & 16,7 & - & $<0,1$
\end{tabular}

Brycoamericus sp. (Rango longitud estandar: $\Delta \mathrm{CP}=4,6-5,2 ; \mathrm{n}=7$ )

\begin{tabular}{lcccc}
\hline Ítem alimenticio & FO & \%FO & D & \%D \\
\hline Resto vegetal & 6 & 85,7 & 6 & 85,7 \\
Formicidae & 1 & 14,3 & 1 & 14,3 \\
Invertebrado & 2 & 28,6 & - & $<0,1$ \\
Semilla & 1 & 14,3 & - & $<0,1$ \\
Baetidae & 2 & 28,6 & - & $<0,1$ \\
\hline
\end{tabular}

Creagrutus cochui (Rango longitud estandar: $\Delta \mathrm{CP}=4,6-5,2 ; \mathrm{n}=4$ )

\begin{tabular}{lcccc}
\hline Ítem alimenticio & FO & \%FO & D & \%D \\
\hline Resto vegetal & 0,5 & 25 & 1 & 25,0 \\
Formicidae & 1,5 & 75 & 3 & 75,0 \\
Invertebrado & 0,5 & 25 & - & $<0,1$ \\
Semilla & 0,5 & 25 & - & $<0,1$ \\
Baetidae & 0,5 & 25 & - & $<0,1$ \\
\hline
\end{tabular}

\begin{tabular}{lcccc}
\hline \multicolumn{4}{l}{ Characidium zebra } & (Rango longitud estandar: $\Delta \mathrm{CP}=6,6-7,1 ; \mathrm{n}=15$ \\
\hline Ítem alimenticio & FO & \%FO & D & \%D \\
\hline Material vegetal & 1 & 20,0 & 0,7 & 13,3 \\
Hydropsychidae & 3,7 & 73,3 & 3,0 & 60,0 \\
Philopotamidae & 0,7 & 13,3 & 0,7 & 13,3 \\
Chironomidae & 0,3 & 6,7 & 0,3 & 6,7 \\
Leptoceridae & 0,3 & 6,7 & 0,3 & 6,7 \\
\hline
\end{tabular}

Hemibrycon sp. (Rango longitud estandar: $\Delta \mathrm{CP}=7-9,5 ; \mathrm{n}=90$ )

\begin{tabular}{|c|c|c|c|c|}
\hline Ítem alimenticio & FO & $\%$ FO & D & $\% D$ \\
\hline Formicidae & 24,0 & 80,0 & 21,0 & 69,7 \\
\hline Resto vegetal & 9,3 & 31,1 & 5,0 & 16,3 \\
\hline Invertebrado & 5,0 & 16,7 & 2,7 & 8,9 \\
\hline Semilla & 2,7 & 8,9 & 1,3 & 4,3 \\
\hline Veliidae & 4,7 & 15,6 & - & $<0,1$ \\
\hline Hydropsychidae & 6,0 & 20,0 & - & $<0,1$ \\
\hline Elmidae & 3,3 & 11,1 & - & $<0,1$ \\
\hline Baetidae & 18,3 & 61,1 & - & $<0,1$ \\
\hline Chironomidae & 6,0 & 20,0 & - & $<0,1$ \\
\hline \multicolumn{5}{|c|}{ Astyanax abramis (Rango longitud estandar: $\Delta \mathrm{CP}=7,6-13,4 ; \mathrm{n}=150$ ) } \\
\hline Ítem alimenticio & FO & $\%$ FO & D & $\% \mathbf{D}$ \\
\hline Escama & 1,4 & 4,7 & 0,2 & 0,66 \\
\hline Semilla & 9,8 & 32,7 & 4,0 & 13,24 \\
\hline Resto vegetal & 12,4 & 41,3 & 13,2 & 43,99 \\
\hline Invertebrado & 7,0 & 23,3 & 1,8 & 5,99 \\
\hline Formicidae & 10,2 & 34,0 & 8,4 & 27,99 \\
\hline Detritus & 11,6 & 38,7 & 0,8 & 2,66 \\
\hline Baetidae & 14,8 & 49,3 & 1,0 & 3,33 \\
\hline Chironomidae & 9,8 & 32,7 & 0,4 & 1,33 \\
\hline Libellulidae & 1,6 & 5,3 & 0,2 & 0,66 \\
\hline Mineral & 4,2 & 20,7 & - & $<0,1$ \\
\hline Leptohyphidae & 5,2 & 17,3 & - & $<0,1$ \\
\hline Ceratopogonidae & 2,4 & 8,0 & - & $<0,1$ \\
\hline Elmidae & 1,2 & 4,0 & - & $<0,1$ \\
\hline Muscidae & 2,4 & 10,0 & - & $<0,1$ \\
\hline Tipulidae & 6,4 & 21,3 & - & $<0,1$ \\
\hline Hydropsychidae & 9,2 & 30,7 & - & $<0,1$ \\
\hline Psychodidae & 2,8 & 11,7 & - & $<0,1$ \\
\hline Tabanidae & 5,4 & 18,0 & - & $<0,1$ \\
\hline Leptoceridae & 2,4 & 8,0 & - & $<0,1$ \\
\hline Acari & 2,0 & 6,7 & - & $<0,1$ \\
\hline Lepidoptera & 2,4 & 8,0 & - & $<0,1$ \\
\hline Hidroptylidae & 1,2 & 4,0 & - & $<0,1$ \\
\hline Simulidae & 2,4 & 8,0 & - & $<0,1$ \\
\hline Bplephariceridae & 1,8 & 6,0 & - & $<0,1$ \\
\hline Philopotamidae & 1,2 & 4,0 & - & $<0,1$ \\
\hline Stratiomyidae & 1,6 & 5,3 & - & $<0,1$ \\
\hline Naucoridae & 6,0 & 20,0 & - & $<0,1$ \\
\hline Leptohplebiidae & 1,2 & 4,0 & - & $<0,1$ \\
\hline Perlidae & 1,2 & 4,0 & - & $<0,1$ \\
\hline
\end{tabular}

El rango de longitud estándar está expresado en $\mathrm{cm}$. Frecuencia de ocurrencia (FO), porcentaje frecuencia de ocurrencia (\%FO), dominancia (D), porcentaje dominancia (\%D). En el caso de Creagrutus cochoui se promedian 2 estaciones (E2, E3); para Characidium zebra 3 estaciones (E1, E2, E3); Hemibrycon sp1. los valores se promedian en 3 estaciones (E2, E3, E4); para Astyanax abramis en 5 estaciones (E2, E3, E4, E5, E6). 

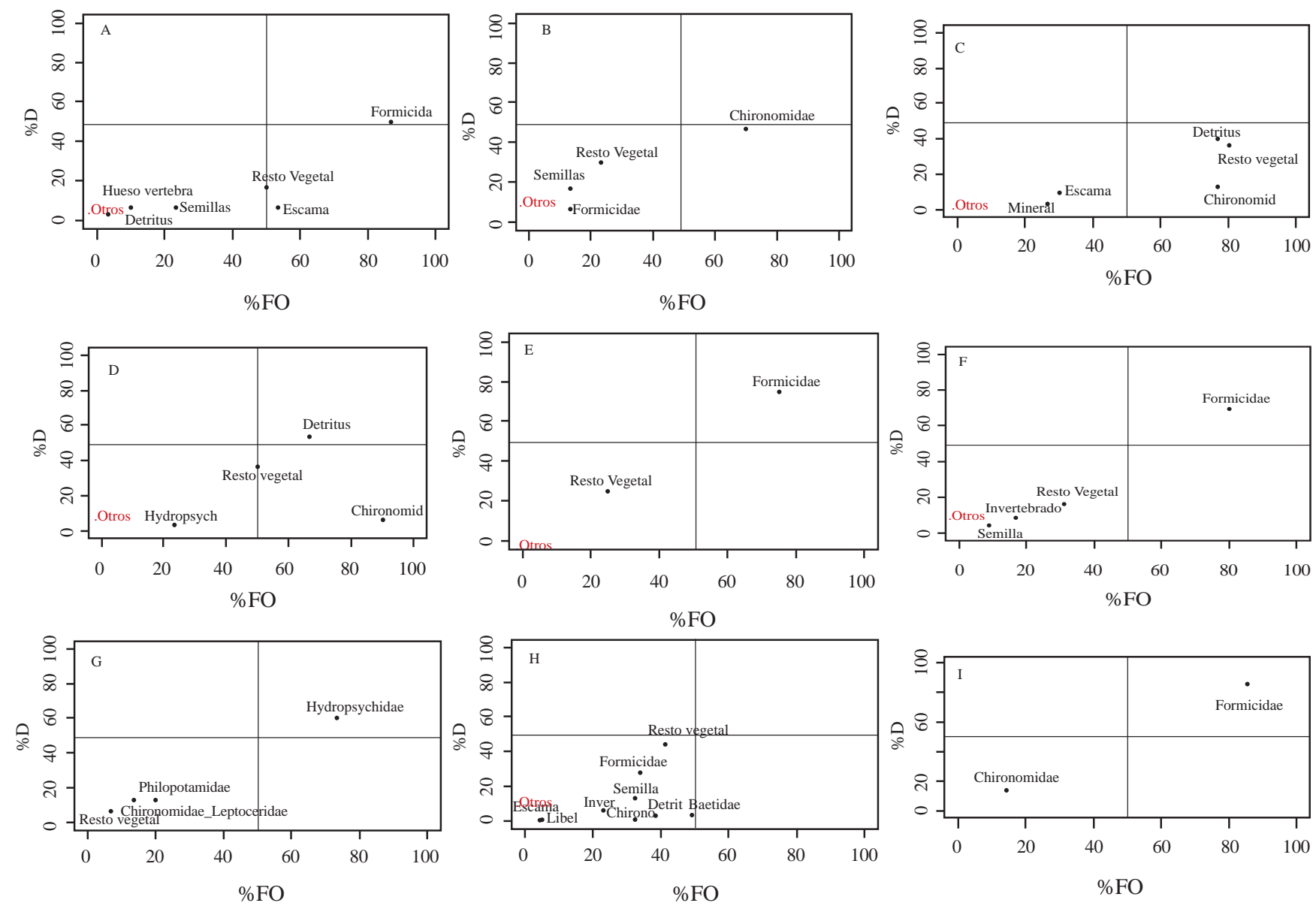

Figura 2. Gráficas de Costello. Porcentaje de frecuencia de ocurrencia (\%FO) y porcentaje de dominancia (\%D) de los ítems alimenticios registrados en Pimelodus blochii (A), Pimelodella cf. cristata (B), Leporinus friderici (C), Leporinus y-ophorus (D), Hemibrycon sp. (E), Creagrutus cochoui (F), Characidium zebra (G), Astyanax abramis (H) Brycoamericus sp. (I).

Leptoceridae que construyen casas con material fino (arena) e Hydropsychidae que elabora refugios con forma de telaraña. Diptera represento el mayor número de familias consumidas $(n=20)$, siendo el recurso de MA más sobresaliente a nivel de riqueza, teniendo en cuenta los valores de frecuencia de ocurrencia. Para Coleoptera se encontró el consumo principal de élmidos, asociados a sustratos rocosos o adheridas en la vegetación riparia (herbáceas identificadas en la estación 6) o raíces sumergidas.

Los tricópteros son organismos con una amplia capacidad de adaptación (Springer et al., 2010), al igual que los dípteros (Courtney \& Merrit, 2008) y coleópteros (White \& Roughley, 2008), aprovechando los diferentes microambientes y recursos disponibles en los ecosistemas acuáticos.

La diversidad de macroinvertebrados acuáticos en un sitio determinado puede estar definida por el sustrato y vegetación ribereña (Vannote, Minshall, Cummins, Sedell \& Cushing, 1980). Con base en las descripciones del área de estudio, las estaciones 1, 2, 3, 4 presentan buenas condiciones de hábitat, con una alta oferta de material alóctono (vegetación ribereña) como fuente de alimento y dominio de sustratos rocosos, que permiten un mayor establecimiento de taxones. En la cuenca baja, el sustrato rocoso empieza a perder dominancia, sobresaliendo las arenas, limos y arcillas, principalmente en la estación 6. De acuerdo al concepto del río continuo (Vannote, et al., 1980), en la cuenca alta y media existe una mayor riqueza de grupos funcionales entre ellos colectores, raspadores, trituradores y depredadores, a diferencia de la cuenca baja, donde estos grupos funcionales se reducen a colectores y depredadores en su mayoría, con una baja colonización en riqueza de MA.

Peces: Los characiformes componen el 34\% de las especies de agua dulce a nivel mundial (Reis, James, Albert \& Bart, 2011) y en la cuenca del Amazonas el 50\% aproximado de las especies reportadas por Géry (1977) pertenecen a este grupo. Este orden se considera dominante en las aguas continentales suramericanas, debido a su gran variedad de formas que les han permitido ocupar un amplio rango de nichos (Galvis, et al., 1997). En la presente investigación se reportan 15 especies, que coinciden con el trabajo desarrollado por Remicio (2014). 
Tabla 5. Hábitos alimenticios en especies ícticas seleccionadas. Se comparan los resultados del proyecto con la literatura.

\begin{tabular}{|c|c|c|c|}
\hline \multirow[t]{2}{*}{ Especie } & \multirow[t]{2}{*}{ Características } & \multirow[t]{2}{*}{ Autor } & \multirow{2}{*}{$\begin{array}{l}\text { Investigación } \\
\text { Habito alimenticio }\end{array}$} \\
\hline & & & \\
\hline \multirow{4}{*}{$\begin{array}{l}\text { Pimelodus blochii } \\
\text { (Valenciennes, 1840) }\end{array}$} & & Hiss, et al., 1978 & \multirow{4}{*}{$\begin{array}{l}\text { Especie insectívora que consume principalmente } \\
\text { hormigas, además de escamas, restos vegetales, } \\
\text { semillas, detritus y macroinvertebrados. }\end{array}$} \\
\hline & Especie omnívora. & Santos, 2000 & \\
\hline & $\begin{array}{l}\text { Especie oportunista } \\
\text { con dieta amplia. }\end{array}$ & $\begin{array}{l}\text { López- Casas \& Jiménez, } \\
2007\end{array}$ & \\
\hline & $\begin{array}{l}\text { Especie que consume } \\
\text { macroinvertebrados } \\
\text { acuáticos y peces. }\end{array}$ & Ramírez \& Pinilla, 2012 & \\
\hline $\begin{array}{l}\text { Pimelodella cf. Cristata } \\
\text { (Miller and Troschel, 1849) }\end{array}$ & $\begin{array}{l}\text { Se alimenta de invertebrados } \\
\text { terrestres, acuáticos y peces. }\end{array}$ & Gutiérrez, 2003 & $\begin{array}{l}\text { Especie omnívora con tendencia invertívora } \\
\text { consumiendo principal de quironómidos. Se } \\
\text { identifican restos vegetales, hormigas y semillas. }\end{array}$ \\
\hline \multirow[t]{2}{*}{$\begin{array}{l}\text { Leporinus friderici } \\
\text { (Bloch, 1974) }\end{array}$} & $\begin{array}{l}\text { Especie omnívora, que consume } \\
\text { material vegetal, insectos } \\
\text { acuáticos y frutos. }\end{array}$ & Gutiérrez, 2003 & \multirow[t]{2}{*}{$\begin{array}{l}\text { Especie omnívora que consume detritus, restos } \\
\text { vegetales, quironómido, escamas y material mineral. }\end{array}$} \\
\hline & Especie omnívora. & Galvis, et al., 2007a & \\
\hline $\begin{array}{l}\text { Leporinus y-ophorus } \\
\text { (Eigenmann 1922) }\end{array}$ & Especie omnívora & Taphorn, 1992 & $\begin{array}{l}\text { Especie con preferencia detritivora, además } \\
\text { consume restos vegetales y macroinvertebrados } \\
\text { acuáticos. }\end{array}$ \\
\hline $\begin{array}{l}\text { Hemibrycon sp1. } \\
\text { (Günter 1984) }\end{array}$ & $\begin{array}{l}\text { Especies del género en su } \\
\text { mayoría son omnívoras. }\end{array}$ & Galvis, et al., 2007b & $\begin{array}{l}\text { Especie insectívora que consume principalmente } \\
\text { hormigas, además restos vegetales. }\end{array}$ \\
\hline $\begin{array}{l}\text { Creagrutus cochoui } \\
\text { (Géry 1964) }\end{array}$ & $\begin{array}{l}\text { Restos de semillas, larvas } \\
\text { y adultos de insectos, restos } \\
\text { de peces. }\end{array}$ & Vari \& Harold, 2001 & $\begin{array}{l}\text { Especie insectívora que consume principalmente } \\
\text { hormigas, además restos vegetales, semillas y } \\
\text { otros invertebrados terrestres. }\end{array}$ \\
\hline \multirow[t]{3}{*}{$\begin{array}{l}\text { Characidium zebra } \\
\text { (Eigenmann 1909) }\end{array}$} & $\begin{array}{l}\text { Pez omnívoro que se alimenta } \\
\text { principalmente de larvas de } \\
\text { simúlidos. }\end{array}$ & Galvis, et al., 2007a & \multirow{3}{*}{$\begin{array}{l}\text { Especie invertívora que consume princiaplmente } \\
\text { trichópteros } \\
\text { de la familia Hydropsychidae. } \\
\text { Además se identificaron tricópteros de la } \\
\text { familia Philopotamidae, dípteros de la familia } \\
\text { Chironomidae y restos vegetales. }\end{array}$} \\
\hline & $\begin{array}{l}\text { Se alimenta de macroinvertebrados } \\
\text { acuáticos, principalmente } \\
\text { ephemerópteros y trichópteros. }\end{array}$ & Pinto \& Uieda, 2007 & \\
\hline & $\begin{array}{l}\text { Consume larvas de dípteros y } \\
\text { efemerópteros. }\end{array}$ & Celtra, et al., 2011 & \\
\hline $\begin{array}{l}\text { Astyanax abramis } \\
\text { (Jenyns 1842) }\end{array}$ & $\begin{array}{l}\text { Especie omnívora o carnívora } \\
\text { (insectos acuáticos) } \\
\text { dependiendo de la talla. }\end{array}$ & Barros, 2004 & $\begin{array}{l}\text { Especie omnívora que consume restos vegetales, } \\
\text { semillas, detritus, macroinvertebrados acuáticos } \\
\text { e invertebrados terrestres. }\end{array}$ \\
\hline $\begin{array}{l}\text { Brycoamericus sp. } \\
\text { (Eigenmann in Eigenmann, } \\
\text { McAtee adn Ward 1907) }\end{array}$ & $\begin{array}{l}\text { Especies de este género se } \\
\text { alimentan de macroinvertebrados } \\
\text { acuáticos y hormigas. }\end{array}$ & Galvis, et al., 1997 & $\begin{array}{l}\text { Especie insectívora que consume hormigas, } \\
\text { además se identifican macroinvertebrados } \\
\text { acuáticos de la familia Chironomidae. }\end{array}$ \\
\hline
\end{tabular}

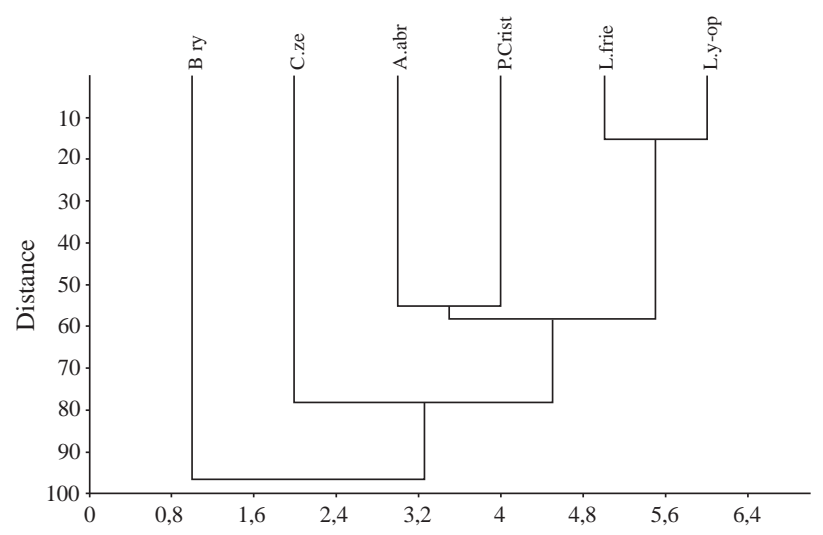

Figura 3. Cluster distancia euclidiana. Las especies se agrupan a partir de los valores obtenidos en los porcentajes de dominancia (\%D). Brycoamericus sp. (Bry), Characidium zebra (Cze), Astyanax abramis (A.abr), Pimelodella cf. cristata (P.Crist), Leporinus friderici (L.frie), Leporinus y-ophorus (L.y-op).
Dentro de los characiformes la familia Characidae representan el 50\% de todas las formas dulceacuícolas suramericanas (Galvis, et al., 1997; Galvis, et al., 2006). Esta familia presenta adaptaciones fisiológicas que le han permitido colonizar diferentes hábitats y mostrar plasticidad alimenticia (Casas, Torres, Lara \& Lozano-Largacha, 2005). Astyanax abramis es la especies más representativa de Characidae en el presente estudio; de acuerdo con Castellanos (2002) Astyanax abramis es frecuente en arroyos selváticos, ocupando principalmente la parte alta de la columna del agua, nadando en grupos de dos o tres individuos en lugares con mayor velocidad de la corriente. Esta especie se colecto en sitios de corriente, remansos y rápidos, identificando grupos aproximados de 3 a 5 individuos, considerándola de acuerdo con la clasificación mencionada por Maldonado-Ocampo, et al. (2005), como una especie fusiforme que se puede adaptar a cambios en el flujo de la corriente. 
Análisis de contenidos estomacales: Los MA son una fuente de alimentación autóctona muy importante para los peces, principalmente sus estados inmaduros (Angermeier \& Karr, 1984) lo que sustenta la importancia relativa de este grupo a nivel de riqueza en las especies analizadas, encontrando de acuerdo con las frecuencias de ocurrencia de 2 hasta 23 item alimenticios relacionados. La influencia depredadora de los peces sobre la comunidad de MA pueden afectar sus abundancias (Flecker, 1984; Gillian, Fraser \& Sabat, 1989; Diehl, 1992; Keller \& Moore, 2000), por lo que es importante pensar a futuro en un análisis que contraste la disponibilidad y consumo en el medio ambiente por parte de las especies de Characiformes y Siluriformes reportadas en el presente trabajo.

Gráficas de Costello (1990): Teniendo en cuenta estos resultados se puede resaltar que a nivel de hábitos alimenticios de las 9 especies evaluadas, sus orígenes son tanto material alóctono como autóctono. Para el primer origen se agrupan 5 especies, Pimelodus blochii, Hemibrycon sp., Brycoamericus sp. y Creagrutus cochoui, cuya principal fuente de alimentación son las hormigas (dominan los formícidos) y Astyanax abramis, especie generalista donde los restos vegetales (R.v) sobresalen en comparación al resto de ítems alimenticios. Para el segundo origen, se agrupan las 4 especies restantes, que dependen de acuerdo con los resultados, de los MA y el detritus; Pimelodella cf. cristata consume principalmente chironómidos, Characidium zebra tricópteros de la familia Hydropsychidae y las especies del género Leporinus sp., dependen principalmente del detritus.

Se podría inferir que el consumo de hormigas se relaciona directamente con el aspecto de "deriva" de invertebrados terrestres y el nivel en el que se encuentran las especies de peces nadando, suponiendo que al caer las hormigas accidentalmente o por una posible migración, de alguna rama o vegetación ribereña, flotan en la superficie, son arrastradas por la corriente y los peces deben moverse muy cerca de la superficie para poder percibirlas. Rezende \& Mazzoni (2006) encuentra una relación significativa entre el consumo de himenópteros por carácidos, teniendo en cuenta su disponibilidad en el medio ambiente y la deriva, al igual Esteves \& Aranha (1999) resaltan la importancia del material de deriva de invertebrados como alimentación, para el establecimiento de peces en arroyos.

Esto también permitiría pensar a futuro la importancia que tiene la estructuración de un ecosistema, en relación medio acuático - medio terrestre, como factores determinantes de la presencia de diferentes especies y la dinámica del flujo de energía a través de una cadena alimenticia, como lo plantea Sullivan, Hossler \& Cianfrani (2015), donde además evalúan el efecto de perturbación sobre el aspecto trófico.

Los restos vegetales que consumen Astyanax abramis, entre ellos, flores, semillas, frutos y hojas, se pueden relacionar directamente con la vegetación ribereña. Vannote, et al. (1980) menciona que la vegetación ribereña es el principal proveedor de alimentos, en zonas donde dominan los procesos heterotróficos, quienes fueron identificados en la cuenca del río Hacha en la mayoría de estaciones.

Las especies que consumen detritus se pueden asociar a fondos o márgenes en el cauce del río. En estos lugares la materia orgánica particulada gruesa (MOPG) empieza a acumularse, para iniciar su proceso de transformación a materia orgánica particulada fina (MOPF), por parte de los hongos, las bacterias y los MA.

En el caso de la comunidad íctica que consume MA, su disponibilidad como alimento está muy relacionada con la heterogeneidad del sustrato y las fuentes de material alóctono disponible (Vannote, et al., 1980; Merrit, et al., 2008).

Solapamiento de nicho: La fauna íctica presenta un nicho trófico muy amplio en comparación con otros vertebrados, existiendo un solapamiento alto a nivel de dietas entre especies (Jaramillo, 2009). Este solapamiento puede estar determinado por la disponibilidad (Mathews, 1998) y abundancia (Eichbaunm, et al., 2008) del alimento, posición habitual de nado de los peces en la columna del agua y ecomorfología. Las especies del género Leporinus habitualmente se encuentran asociadas a barrancos con dominancia de pastizales o arboles (Galvis et al., 2007 a, b), características similares en las que fueron encontrados en esta investigación, identificando un solapamiento de hábitat $\mathrm{y}$ de dietas entre esta dos especies.

\section{Conclusiones}

De los MA, la clase Insecta fue la categoría taxonómica dominante y en términos de órdenes los tricópteros presentaron la mayor riqueza de géneros dentro de esta comunidad, indicando un mayor nivel de adaptación y aprovechamiento de los recursos en este ecosistema.

Las estaciones 1, 2, 3 y 4 relacionadas con la cuenca alta, media y poca a mediana intervención antrópica, presentaron los mejores valores de diversidad de MA, disminuyendo significativamente en las estaciones 5 y 6 , quienes se ubican en la cuenca baja, con alta intervención.

Con respecto a los peces, los Characiformes dominaron en la cuenca del río Hacha, siendo la familia Characidae la que presentó mayor número de especies, sobresaliendo Astyanax abramis con mejor distribución y abundancia en la cuenca del río Hacha.

A partir del análisis de contenido estomacal se identificaron 2 grupos tróficos: especies generalistas omnívoras que contienen MA como Astyanax abramis, Leporinus frieredici y Pimelodella cf. Cristata, esta última con tendencia a especialista (invertívora). Especies especialistas que pueden o no contener MA, como Pimelodus blochii, Hemibricon sp. Brycoareicus sp. y Creagrutus cochoui, este último identificado como insectívora, consumiendo principalmente hormigas (Formicidae). Characidium zebra es la única especie especialista que depende del consumo principal de macroinvertebrados acuáticos. 
Astyanax abramis y Characidium zebra están más relacionadas con el consumo de macroinvertebrados acuáticos. La primera, a nivel de riqueza de taxones consumidos dentro de la dieta omnívora y la segunda como principal fuente de alimentación.

Con respecto al solapamiento de nicho, Leporinus friederici y Leporinus y-ophorus presentaron el mayor similaridad dentro de la comunidad estudiada, compartiendo hábitat y dieta en la estación 6, ubicada en la parte baja de la cuenca.

\section{Agradecimientos}

A Socios para la Conservación de la Amazonía Colombiana, por su apoyo económico y académico en el desarrollo de la presente investigación. A la Universidad de la Amazonía por su colaboración en el préstamo de equipos e instalaciones para la revisión y análisis de datos.

\section{Conflicto de intereses}

Los autores declaran no tener conflictos de intereses.

\section{Referencias}

Angermeier, P. \& Kare, J. 1984. Fish communities along environmental gradients in a systems of tropical streams. Environmental Biology of Fishes. 9: 117 - 135.

Aten-García V. J., Kergelen-Durango, E., Cura-Dorado, E., Rosado Carcamo, R., Vallejo, A., Valderrama, M. 2005. Régimen alimentario de siete especies ícticas en el embalse de la hidroeléctrica Urrá (Córdoba, Colombia). Revista Científica de la Facultad de Medicina Veterinaria y Zootecnia. 10 (2): 614 - 622.

Amundsen, P. A, Gabler, H. M. \& Staldvik, F. J. 1996. A new approach to graphical analysis of feeding strategy from stomach contents data-modification of the Costello (1990) method. Journal of Fish Biology. 48: 607 - 614.

Barros S. E. 2004. Alimentación de Astyanax abramis (Characiformes: Characidae) en el embalse CabraCorral, Salta, Noroestede Argentina. Revista Aquatica. 20: 88 - 96.

Bennemann, T., Casatti, L. \& Cristiane de Oliveira, D. 2006. Alimentação de peixes: proposta para análise de itens registrados em conteúdos gástricos. Biota Neotropica. 6 (2): 1 - 8.

Bleeker, P. 1862. Atlas ichthyologique des Indes Orientales Néêrlandaises, publié sous les auspices du Gouvernement colonial néêrlandais. Tome II. Siluroïdes, Chacoïdes et Hétérobranchoïdes. F. Muller, Amsterdam. 1 - 112, Pls. 49 - 101.

Bloch, M. E. 1974. Naturgeschichte der ausländdischen Fische. Vol 8. Berlin. iv + 174: 361 - 396.

Carpenter S. R., Kitchell, J. F., Hodgson, J. R., Cochran, P. A., Elser, J. J., Elser, M. M., Lodge, D. M., Kretchmer, D., He, X., Von Ende, C. N. 1987. Regulation of lake primary productivity by food web structure. Ecology. 68: 1863-1876.

Casas, A. J, Torres, O. C., Lara, R. T. \& Lozano-Largacha, Y. 2005. Composición y diversidad íctica en la quebrada Chaparraido, sistema hídrico del medio Atrato, ChocóColombia. Revista Institucional, Universidad Tecnológica del Chocó D. L. C. 22: 56-62.
Castellanos, C. 2002. Distribución espacial de la comunidad de peces en una quebrada de aguas negras amazónicas, Leticia, Colombia (Tesis de pregrado). Biología, Universidad Nacional de Colombia. Bogotá. Colombia.

Celtra, M., Rodríguez Rondineli, G. \& Pereira Souza, U. 2011. Compartilhamento de recursos por duas espécies de peixes nectobentônicas de riachos na bacia do rio Cachoeira (BA). Biota Neotropica. 11 (2): 87-95.

Costello, M. J. 1990. Predator feeding strategy and prey importance: a new graphical analysis. Journal. Fish Biology. 36: 261-263.

Courtney, G. W. \& Merritt, R. W. 2008. Aquatic Diptera, Part One. Chapter 22. In: R. W. Merritt, K. W. Cummins, M. B. Berg. (ed). Introduction to the aquatic insects of North America, 4th ed. (pp. 687 - 690). Kendall: Hunt publishing Company. USA

Cuvier, G. 1816. Le Règne Animal distribué d'après son organisation pour servir de base à l'histoire naturelle des animaux et d'introduction à l'anatomie comparée. Les reptiles, les poissons, les mollusques et les annélides. Edition 1. 2: i-xviii+1-532, Pls. 9-10, in v. 4.

Cuvier, G. 1819. Sur les poissons du sous-genre Hydrocyon, sur deux nouvelles espèces de Chalceus, sur trois nouvelles espèces du Serrasalmes, et sur l'Argentina glossodonta de Forskahl, qui est l'Albula gonorhynchus de Bloch. Mémoires du Muséum National d'Histoire Naturelle, Paris (N. S.) (Série A) Zoologie. 5: 351-379, Pls. 26-28.

Cuvier, G. \& Valenciennes A. 1840. Histoire naturelle des poissons. Tome quinzième. Suite du livre dix-septième. Siluroïdes. 15: i-xxxi + 1-540, Pls. 421-455.

Cuvier, G. \& Valenciennes, A. 1850. Histoire naturelle des poissons. Tome vingt-deuxième. Suite du livre vingt-deuxième. Suite de la famille des Salmonoïdes. Table générale de l'Histoire Naturelle des Poissons (pp. 1-91). 22: i-xx+1 p. + 1-532 + 1-91, Pls. 634-650.

Diehl, S. (1992). Fish predation and Benthic community structure: Ther role of omnivory and habitat complexity. Ecology. 5: 1646-1661.

Domínguez E. \& Fernández, H. R. 2009. Macroinvertebrados bentónicos sudamericanos. Sistemática y biología. ( $2^{\mathrm{da}}$ edición). Tucuman: Fundación Miguel Lilio. Argentina.

Eichbaum Esteves, K., Pinto Lobo, A. V. \& Renó Faria, M. D. 2008. Trophic structure of a fish community along environmental gradients of a subtropical river (Paraitinga River, Upper Tiete^ River Basin, Brazil). Hydrobiologia. 598: 373-387.

Eigenmann, C. H., McAtee W. L., Ward, D. P. 1907. On further collections of fishes from Paraguay. Annals of the Carnegie Museum. 4 (2): 110-157, Pls. 31-45.

Eigenmann, C. H. 1909. Reports on the expedition to British Guiana of the Indiana University and the Carnegie Museum, 1908. Report no. 1. Some new genera and species of fishes from British Guiana. Annals of the Carnegie Museum. 6 (1): 4-54.

Eigenmann, C. H. 1911. Descriptions of two new tetragonopterid fishes in the British Museum. Annals and Magazine of Natural History (Series 8). 7 (38): 215-216.

Eigenmann, C. 1922. The fishes of western South America, part I. The Fresh-water fishes of northwestern South America, including Colombia, Panama and the Pacific slopes of Ecuador and Peru, together with an appendix upon the fishes of the Río Meta in Colombia. Memories of Carnegie Museum. 9 (1): 1-346. 
Eigenmann, C. H. \& Allen, W. R. 1942. Fishes of Western South America. I. The intercordilleran and Amazonian lowlands of Peru. II.- The high pampas of Peru, Bolivia, and northern Chile. With a revision of the Peruvian Gymnotidae, and of the genus Orestias. University of Kentucky. i-xv $+1-494$, Pls. 1-22.

Epler J. H. 1995. Identification manual for the larval Chironomidae (Diptera) of Florida. Tallahassee: Florida Department of Environmental Protection. USA

Epler J. H. 1996. Identification manual of the water beetles of Florida (Coleoptera: Dryopidae, Dysticidae, Elmidae, Gyrinidae, Haliplidae, Hydraenidae, Hydrophilidae, Noteridae, Psephenidae, Ptilodactylidae, Scirtidae). Tallahassee: Florida Department of Environmental Protection. USA

Esteves, K. E. \& Aranha, M. R. 1999. Ecologia trófica de peixes de riachos. In: E. P. Caramashi, R. Mazzoni, Peres-Neto, P. R. (ed). Ecologia de peixes de riachos. (pp 157-182). Série Oecologia Brasiliensis. 7.

Flecker, A. S. 1984. The effects of predation and detritus on the structure of a stream insect community: a field test. Oecologia. 64: 300-305.

Flecker, A.S. 1992. Fish predation and the evolution of invertebrate drift periodicity: evidence from Neotropical streams. Ecology. 73: 438-448.

Galvis, G., Mojica, J. I. \& Camargo, J. Y. M. 1997. Peces del Catatumbo. Asociación Cravo Norte. D’Vinni Editorial Ltda. Bogotá.

Galvis G, Mojica, J. I., Duque, S. R., Castellanos, C., SánchezDuarte, P., Arce, M., Gutiérrez, A., Jiménez, L. F., Santos, M., Vejarano, S., Arbeláez, F., Prieto, E. \& Leiva, M. 2006. Peces del medio Amazonas, Región Leticia. Serie de Guías Tropicales de campo No 5. Conservación Internacional. Bogotá: Editorial Panamericana, formas e impresos.

Galvis G., Mojica, J. I., Provenzano, F., Lasso, C. A., Taphorn, D. C., Royero, R., Castellanos, C., Gutiérrez, A., Gutiérrez, M. A., López, Y., Mesa, L. M., P., Sánchez Duarte \& Cipamocha, C. A. 2007a. Peces de la Orinoquía colombiana con énfasis en especies de interés ornamental. INCODER y Universidad Nacional de Colombia.

Galvis, G., Sánchez-Duarte, P., Mesa-Salazar, L. M., LopezPinto, Y., M. A., Gutiérrez-E, Gutiérrez-Cortés, Á., Leiva-Castaño, M. \& Castellano-Castillo, C. 2007b. Peces de la Amazonía Colombiana con Énfasis en especies de interés ornamental. INCODER, Universidad Nacional de Colombia y SINCHI.

Géry, J. 1964. Poissons characoïdes nouveaux ou non signalés de I’llha do Bananal. Brésil. Vie Milieu Suppl. 17: 447-471.

Géry, J. 1977. Characids of the World. Neptune City: T.F.H. Publications. USA

Gregory, J. D \& Maldonado-Ocampo, J. A. 2006. Peces de la zona hidrogeográfica de la Amazonia, Colombia. Instituto de Investigaciones en Recursos Biológicos Alexander von Humboldt. Biota Colombiana. 7 (1): 55-94.

Gillian, J. F., Fraser, F. D. F. \& Sabat, A. M. 1989. Strong Effects of Foraging Minnows on a Stream Benthic Invertebrate Community. Ecology. 70 (2): 445-452.

Günter, A. 1864. Catalogue of the fishes in the Brithish Museum, vol 5. Catalogue of the Physostomi, containing the families Siluridae, Characidinae, Haplochitonidae, Sternoptychidae, Scopelidae, Stomiatidae in the collection of the Brithish Museum. Trustees, London. xxii+455.
Gutiérrez, A. 2003. Análisis de algunos aspectos tróficos y reproductivos de la comunidad de peces de un caño de aguas negras amazónicas en cercanías de Leticia (Amazonas, Colombia) (Tesis de Pregrado). Departamento de Biología, Facultad de Ciencias, Universidad Nacional de Colombia.

Hahn, N. S., Fugi, R., De Almeida, V. L. L., Russo, M. R. \& Loureiro, V. E. 1997. Dieta e atividade alimentar de peixes do reservatorio de Segredo. In: A. A. Agostinho \& L. C. Gomes (ed). Reservatório de Segredo: bases ecológicas para o manejo. (pp 142-162). Maringá: Nupelia, Editora da Universidade Estadual de Maringá (EDUEM).

Hammer, Ø., Harper, D.A.T., Ryan, P.D. 2001. PAST: Paleontological Statistics Software Package for Education and Data Analysis. Paleontología Electrónica 4(1). http:// paleo-electronica.org/2001_1/past/issue1_01.htm.

Heckman, W. C. 2008. Encyclopedia of South America aquatic insects: Odonata-Zigoptera. Ilustrated keys to know families, genera and species in South America. Olympia: Springer editorial. USA

Hiss, J., Shirley, K. \& Aristizabal, W. 1978. La pesca en la represa de Prado, Tolima, 1974-1978. Bogotá: Publicación del Cuerpo de Paz. Bogotá.

Hyslop, E. J. 1980. Stomach contents analysis. A review of methods and their applications. Journal. Fish Biology. 17: 411-429.

Isbrücker, I. J. H. 1980. Classification and catalogue of the mailed Loricariidae (Pisces, Siluriformes). Verslagen en Technische Gegevens, Instituut voor Taxonomische Zoöogie, Universiteit van Amsterdam No. 22: 1-181.

Jaramillo Londoño, A. 2009. "Estudio de la biología trófica de cinco especies de peces bentónicos de la costa de Cullera, Relaciones con la acumulación de metales pesados (Tesis Doctoral). Universidad Politécnica de Valencia, Departamento de Ingenieria Hidráulica y Medio Ambiente.

Jenyns, L. 1842. Fish. In: The zoology of the voyage of H. M. S. Beagle, under the command of Captain Fitzroy, R. N., during the years 1832 to 1836. London: Smith, Elder, and Co. Issued in 4 parts. i-xvi + 1-172, Pls. 1-29.

Kasprzak, P., Benndorf, J., Mehner, T. \& Koschel, R. 2002. Biomanipulation of lake ecosystems: research progress, water quality and fisheries management. Freswater. Biology. 47 (12): 2277-2281.

Kner, R. 1854. Die Hypostomiden. Zweite Hauptgruppe der Familie der Panzerfische. (Loricata vel Goniodontes). Denkschriften der Kaiserlichen Akademie der Wissenschaften in Wien, Mathematisch-Naturwissenschaftliche Classe. 7: 251-286, separate 1 - 36, Pls. 1-5

Kner, R. 1858. Beiträge zur Familie der Characinen. Sitzungsber. Akad. Wiss. Wien. 30 (13): 75-80.

Keller, T. A. \& Moore, P. A. 2000. Context-specific behavior: crayfish size influences crayfish-fish interactions. Journal National America Benthology Society. 19 (2): 344-351.

Klemm, D. J. 1995. Identification guide to the freshwater leeches (Annelida: Hirudiena) of Florida and other southern state. Tallahasee: Department of Environmental Protection, Division of Water Facilities. USA

Linnaeus, C. 1758. Systema naturae per regna tria naturae, secundum classes, ordines, genera, species, cum characteribus, diferentiis, synonomis, locis. Editio decima, reformata. Tomus I. Holmiae. ii + 824 p. 
López-Casas, S. \& Jiménez-Segura, L. F. 2007. Reproducción y hábitos alimenticios del nicuro, Pimelodus blochii (Valenciennes, 1840) (pisces: pimelodidae), en la ciénaga de Cachimbero, río Magdalena, Colombia. Actualidades Biológicas. 29 (87): 199-207.

López-Erazo, I. S., Gaspar, S. \& Peláez-Rodríguez, M. 2012. Aplicación de índices bióticos para la evaluación de la calidad del agua en un río Andino Amazónico. Momentos de Ciencia. 9 (2): 106-112.

McCafferty, W. P. 1981. Aquatic entomology the fishermens and ecologists illustrated guide to insect and their relatives. Boston: Science Books International.

Maldonado-Ocampo, J. A., Ortega-Lara, A., Usma Oviedo, J. S., Galvis Vergara, G., Villa-Navarro, F. A., Vásquez Gamboa, L., Prada-Pedreros, S. \& Ardila Rodríguez, C. 2005. Peces de los andes de Colombia, guía de campo. Instituto de investigaciones de recursos biológicos “Alexander von Humboldt". Bogotá.

Marrero, C. 1994. Métodos para cuantificar contenidos estomacales en peces. Universidad Nacional Experimental de los Llanos Occidentales Ezequiel Zamora. Caracas: Centauro.

Masiac, Y., Guilbot, R. \& Albouy, V. 2006. El fascinante mundo de los artrópodos, "insectos, mariposa, arañas y mariquitas. Barcelona: Editorial De Vecchi.

Meijer M. L., De Boois, I., Scheffer, M., Portielje, R. \& Hosper H. 1999. Biomanipulation in shallow lakes in the Netherlands: an evaluation of 18 case studies. Hydrobiologia. 408/409: 13-30.

Merrit, R.W., Cummins, K.W. \& Berg, M.B. 2008. An Introduction to the Aquatic Insects of North America (4th ed). Kendall: Hunt Publishing Company.

Mojica-C, J. I. 1999. Lista Preliminar de las especies de peces dulceacuícolas de Colombia. Revista de la Academia Colombiana de Ciencias Exactas, Físicas y Naturales. Vol 23, suplemento especial.

Müller, J. \& F. H. Troschel. 1849. Fische. Pp. 618-644, In: Schomburgk, R. Reisen in Britisch-Guiana in den Jahren 1840-44. Im Auftrag Sr. Mäjestat des Königs von Preussen. Versuch einer Fauna und Flora von Britisch-Guiana. Leipzig, Verlagsbuchhanlung von J.J. Weber

Muñoz, I., Romaní, A. M., Rodríguez-Capítulo, A., González Esteban, J. \& García-Berthou, E. 2009. Relaciones tróficas en el ecosistema fluvial, Capítulo 19. En: A. Elosegi \& S. Sabater (ed). Conceptos y técnicas en ecología fluvial. (pp 345 - 366). Fundación Banco Bilbao Vizcaya Argentaria (BBVA).

Olaya-Nieto, W. C., Soto-Fernández, P., Barrera-Chica, J. 2009. Hábitos alimentarios de la mayupa (Sternopygus macrurus Bloch \& Schneider, 1801) en el río Sinú, Colombia. Revista Cientifica de la Facultad de Medicina Veterinaria y Zootecnia. 14 (3): 1787-1795.

Olaya-Nieto, W. C., Arellano-Padilla, J. J. \& MartínezGonzález, A. L. 2012. Hábitos alimentarios del barbudo de piedra (Ariopsis sp.) en el Río Sinú, Colombia: Acta biológica Colombiana. 17 (1): 117-128

Ortaz M., Martín, R. \& López-Ordaz, A. 2011. Variación espacial y temporal en la composición de peces invertíboros en un río Neotropical, Venezuela. Revista Biología Tropical. 59 (3): 1217-1231.

Pescador, M.L., Rasmussen, A. K. \& Harris, S. C. 2002. Identification manual for the Caddislfy (Trichoptera) Larvae of Florida. Tallahasee: Florida Department of Environmental Protection Division of Water Resource Management. USA
Pescador, M.L. \& Barton, A.R. 2004. Guide to the Mayfly (Ephemeroptera) Nymphs of Florida. Tallahasee: Florida Department of Environmental Protection Division of Water Resource Management. USA

Pinto, T. L. F. \& Uieda, V. S. 2007. Aquatic insects select as food for fishes of a tropical stream: are there spatial and seasonal differences in their selectivity? Acta Limnologica. Brasiliensia. 19 (1): 67-78.

Quirós, R. \& Boveri, M. B. 1999. Fish effects on reservoir trophic relationhip. In: J. G. Tundisi \& M. Straškraba (ed). Theoretical reservoir ecology and its applications. (pp 529546). Backhuys: Academia Brasileira de Ciencias.

Ramírez, A. \& Viña, G. 1998. Limnología Colombiana: Aportes a su conocimiento y estadísticas de análisis. Bogotá: Editorial Panamericana.

Ramírez, A. \& Pinilla, G. 2012. Hábitos alimentarios, morfometría y estados gonadales de cinco especies de peces en diferentes períodos climáticos en el río Sogamoso (Santander, Colombia). Acta Biológica Colombiana. 17 (2): 241-258.

Reis, R., James, S. Albert. \& Bart, H. 2011. Species Richness and Cladal Diversity Chapter 5, Historical Biogeography of Neotropical Freshwater Fishes. Bekerley: University of California Press.

Remicio J. H. 2014. Estructura del orden Characiformes a través del gradiente altitudinal en la cuenca del río Hacha, Florencia-Caquetá (Tesis de Maestría). Maestría en Ciencias Biológicas. Universidad de la Amazonia. Florencia. Colombia.

Renó Braga, R., Bornatowski, H. \& Simões Vitule, J. R. 2012. Feeding ecology of fishes: an overview of worldwide publications. Review in Fish Biology and Fisheries. 22: 915-929.

Rezende C. F. \& Mazzoni, R. 2006. Disponibilidade e uso de recursos aloctones por Bryconamerius microcephalus (Miranda-Ribeiro) (Actinopterygii, Characidae), no corrego Andorinha, Ilha Grande, Rio de Janeiro, Brasil. Revista Brasileira de Zoologia. 23 (1): 218-222.

Roche, K. \& Rocha, O. 2005. Ecologia Trófica de Peixes, con ênfase na planctivoria em ambientes lênticos de agua doce no Brasil. Sao Carlo: RiMa Editora.

Roldán, G. 1988. Guía para el estudio de los macroinvertebrados acuáticos del Departamento de Antioquia. Bogotá: Editorial Presencia Ltda.

Roldán, G. 1992. Fundamentos de Limnología Neotropical. Medellín: Editorial Universidad de Antioquia.

Roldán G., Posada, J. \& Gutierrez, J. 2001. Estudio limnológico de los recursos hídricos del Parque de Piedras Blancas. Bogotá: Academia Colombiana de Ciencias Exactas, Físicas y Naturales.

Roldán, G. \& Ramírez, J. 2008. Fundamentos de Limnología Neotropical (2a ed.). Medellín: Editorial Universidad de Antioquia.

Román-Valencia C. 2007. Una nueva especie de pez del género Hemibrycon (characiformes: characidae) del alto río Atrato, noroccidente de Colombia. Caldasia. 29 (1): 121-131.

Rubio, E. A. 2007. Introducción a los Peces Dulceacuícolas de Colombia, una introducción para estudiantes de ictiología. Cali: Facultad de Ciencias Exactas y Naturales, Universidad del Valle. 
Sánchez, R. M., Galvis, G. \& Victoriano, P. F. 2003. Relación entre características del tracto digestivo y los hábitos alimentarios de peces del río Yucao, sistema del Río Meta (Colombia). Gayana. 67 (1): 75-86.

Sanabria, A. I, Daza, P. V. \& Beltran I. C. 2007. Peces de la Amazonia Colombiana con Énfasis en Especies de Interés Ornamental. Ministerio de Agricultura y Desarrollo Rural. INCODER. Bogotá: Universidad Nacional de Colombia.

Santos M. 2000. Aspectos ecológicos de la fauna íctica dominante en la lagu na de Yahuarcaca, Leticia (Amazonía Colombiana) (Tesis de Pregrado). Departamento de Biología. Facultad de Ciencias, Universidad Nacional de Colombia.

Spix, J. B. von \& Agassiz, L. 1829. Selecta genera et species piscium quos in itinere per Brasiliam annos MDCCCXVIIMDCCCXX jussu et auspiciis Maximiliani Josephi I.... colleget et pingendso curavit Dr J. B. de Spix. Monachii. Part 1: i-xvi + i-ii + 1-82, Pls. 1-48; Part 2: 83-138, Pls. 49-101.

Springer, M., Ramirez, S., Hanson, P. 2010. Macroinvertebrados de agua dulce de Costa Rica I. Revista Biología Tropical. 58.

Steindachner, F. 1876. Ichthyologische Beiträgue (V). Sitzungsber. Akad. Wiss. Wien. 74: 49-240, pls. 1-15.

Sullivan, S. M., Hossler, K., Cianfrani, C. M. 2015. Ecosystem Structure Emerges as a Strong Determinant of Food-Chain Length in Linked Stream-Riparian Ecosystems.

Taphorn, D. 1992. The characiform fishes of the Apure river drainage, Venezuela. Biollania. 4: 1-537.

Torres, Y., Roldán, G., Asprilla, S., Rivas, T. S. 2006. Estudio preliminar de algunos aspectos ambientales y ecológicos de las comunidades de Peces y Macroinvertebrados acuáticos en el Río Tutunendo, Chocó, Colombia. Revista Academia Colombiana de Ciencias. 30 (114): 67-76.

Tschudi, J. J. von. 1846. Ichthyologie. Pp. ii-xxx + 1-35, Pls. 1-6. In: Untersuchungen über die Fauna Peruana. Scheitlin \& Zollikofer, St. Gallen. 1844-46, in 12 parts. Parts 1-2 issued in 1844, 3-5 in 1845, 6-12 in 1846. Overall pp. 1-693. Fishes evidently all in 1846.

Vannote R. L., G. Minshall, W., Cummins, K. W., Sedell, J. R. \& Cushing, C. 1980. The river continuum concept. Canadian Journal of Fisheries and Aquatic Sciences. 37: 130-137.

Vari, R. \& Harold A. S. 2001. Phylogenetic study of the neotropical fish genera Creagrutus Günther and Piabucina Reinhardt (Teleostei: Ostariophysi: Characiformes), with a revision of the cis-andean species. Smithson. Contribution Zoology. 613.

Voshell, J.R. 2002. A guide to common freshwater invertebrates of North America. Blacksburg: The McDonald y Woodward Publishing Company.

White D. S. \& Rougley R. E. 2008. Aquatic Coleoptera. Chapter 20. In: R.W. Merritt, K.W. Cummins \& M.B. Berg (ed). Introduction to the aquatic insects of North America (4th ed). (pp 571-671). Kendall: Hunt publishing Company.

Zamudio, J., Urbano-Bonilla, A., Maldonado-Ocampo, J. A., Bogota-Gregory, J. D. \& Cortes-Millan, G. A. 2008. Hábitos Alimentarios de diez especies de peces del piedemonte del departamento del Casanare, Colombia. Dahlia (Revista Asociación. Colombiana de Ictiólogos). 10: 43-55. 